\title{
FERMILAB ELECTRON COOLING PROJECT: COMMISSIONING OF THE 5 MEV RECIRCULATION TEST SET-UP
}

\author{
A.Burov, A.C. Crawford, K.Carlson, V.Dudnikov, T. Kroc, J. Leibfritz, M. McGee, S. Nagaitsev, \\ G. Saewert, C.W. Schmidt, A.Shemyakin*, A. Warner, FNAL ${ }^{\bullet}$, Batavia, IL 60510, USA
}

\section{Abstract}

An important part of the Fermilab's Recycler Electron Cooling (REC) project [1] is a recirculation test which is performed using a 5-MV electrostatic accelerator, a Pelletron, with two tubes and a simplified beam line with one 180-degree bend. The main goal of the test is to demonstrate stable operation of a 4.4-MeV, 0.5-A DC electron beam. The paper describes the set-up and the early experimental results.

\section{INTRODUCTION}

Electron cooling in the Recycler ring needs a highpower DC beam. The beam generation scheme employs an electrostatic accelerator, Pelletron, in a so-called recirculation regime: the beam is accelerated down one tube of the Pelletron, passes through the beam line at ground, and returns to the high voltage terminal through the second tube. The great advantage of the scheme is a low dissipated power (several $\mathrm{kW}$ ) while the beam power in the beam line is more than $2 \mathrm{MW}$. The scheme has been successfully tested at the electron energy of $1.2-1.5$ $\mathrm{MeV}$ [2]. To prepare a beam generation system for a future full-scale electron cooling device, an experimental set-up with a simplified beam line has been manufactured, installed and is now being commissioned at Fermilab. The main goal of the experiment is demonstration of a stable operation of an electron beam with parameters listed in Table 1.

Table 1: Electron beam parameters

\begin{tabular}{|c|c|c|}
\hline Electron kinetic energy & 4.36 & $\mathrm{MeV}$ \\
\hline Electron Beam Current & 0.5 & A \\
\hline Terminal Voltage Ripple & 500 & V (FWHM) \\
\hline Cathode Radius & 2.5 & $\mathrm{~mm}$ \\
\hline Magnetic Field at Cathode & $\cdot 600$ & $\mathrm{G}$ \\
\hline
\end{tabular}

\section{SET-UP DESCRIPTION}

The mechanical schematic of the set-up is shown in Fig.1. A 5-MV Pelletron is produced by National Electrostatic Corp. (NEC) [3]. The Pelletron tank is filled with $\mathrm{SF}_{6}$ gas under 5 -6 atm for high voltage insulation. The Pelletron column is formed by six aluminum discs separated by ceramic posts. The lower four, called separator boxes, have potentials from $U_{0} / 5$ to $4 U_{0} / 5$, where $U_{0}$ is the potential of the fifth disc, the terminal. The terminal has an attached shell where the last disc, the

\footnotetext{
*Corresponding author. E-mail:shemyakin@fnal.gov

- Work supported by the U.S. Department of Energy under contract No. DE-AC02-76CH03000.
}

deck, is installed.

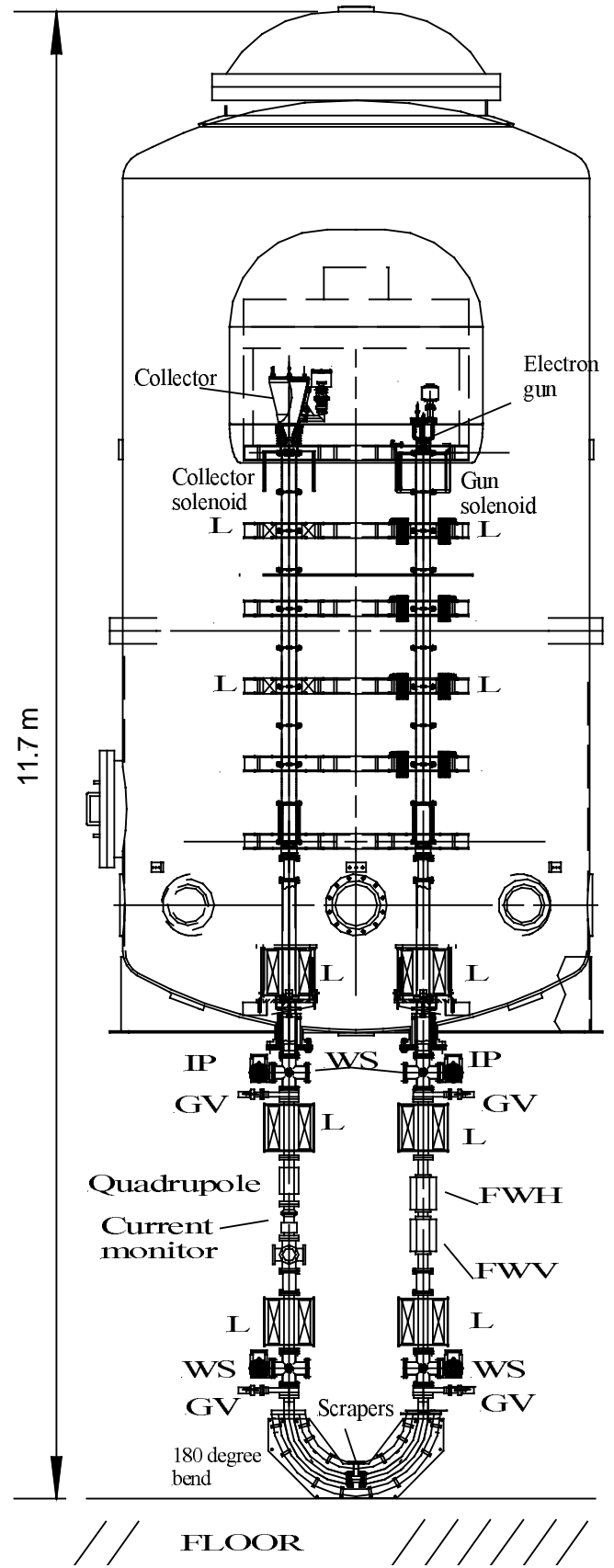

Figure 1: Mechanical schematic of the U-bend test stand. Symbols denote, correspondingly: IP- ion pump, L- lens, GV- gate valve, WS- wire scanners, FWH and FWVflying wires. 
Each disc is equipped with an electrical generator, powered by a single rotating insulated rod, and by a control unit, communicating with the control system at ground through a fiber link. Both accelerating and decelerating tubes consist of ten identical metal-ceramic modules. Every second module is connected to a separation box; the tops of the tubes and the gun and collector anodes have the terminal potential; the cathode is electrically connected to the deck.

Two "chains" charging the high voltage terminal can supply current, $I_{\text {chain }}$, up to $300 \mu \mathrm{A}$. The total current through the column and tube resistive dividers, $I_{r e s}$, at the nominal energy is about $120 \mu \mathrm{A}$. Therefore, up to $180 \mu \mathrm{A}$ can be used to compensate the current loss $\delta I$.

The DC and AC components of the terminal voltage are measured by a generating voltmeter (GVM) and by two capacitive pickups (CP), correspondingly. Since the GVM was calibrated at $U_{0}=100 \mathrm{kV}$, the absolute precision of voltage measurements at a multi- MV level is estimated to be $2 \%$. Using GVM and CP signals, the voltage stabilization system adjusts the corona current, $I_{\text {needle }}$, drawn from needles installed at the terminal so that the voltage is kept at an equilibrium:

$$
C \frac{d U_{0}}{d t}=I_{\text {chain }}-I_{\text {res }}-I_{\text {needle }}-\delta I-I_{\text {cor }}=0
$$

where $\mathrm{C}=330 \mathrm{pF}$ is the terminal capacitance and $I_{\text {cor }}$ is the parasitic corona current from the terminal. If either of two last terms increases, the control system decreases $I_{\text {needle }}$ by the same value to keep the voltage stable. After proper conditioning (see section 3.1), $I_{\text {cor }}$ fluctuates by $1-2 \mu \mathrm{A}$, and the change in the needle current is used as a measure of a the beam-related loss (section 3.2).

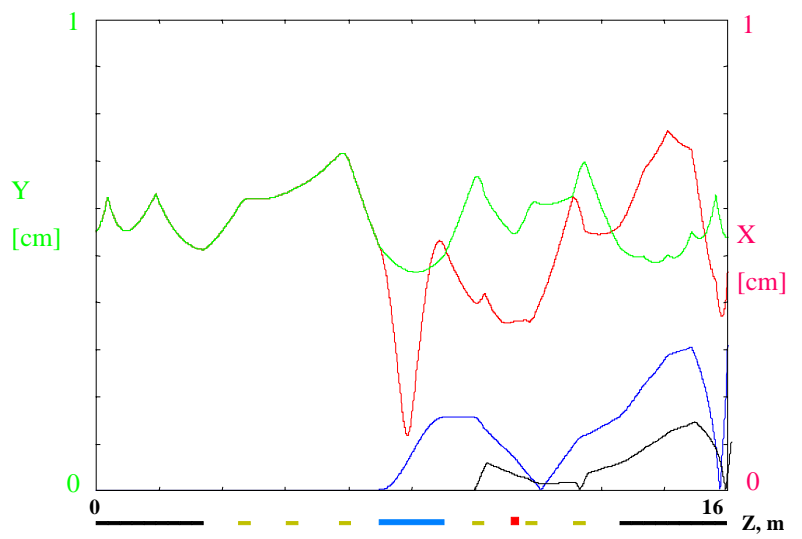

Figure 2. Beam envelope for the beam energy of 4.357 $\mathrm{MV}$, beam current of $1 \mathrm{~A}$, and the magnetic field at the cathode of $600 \mathrm{G}$. Two lower curves are dispersions.

The upper modules of both tubes are immersed in solenoids protruding from the terminal. A short solenoid around the gun generates a longitudinal magnetic field on the cathode. In the tubes, the beam is focused by solenoidal lenses installed in each separator box.

Maximum power dissipated inside the tank is $25 \mathrm{~kW}$. The tank is cooled by a flow of SF6 gas, which is watercooled in an outside heat exchanger.
The beam line optics consists of six solenoidal lenses, an air-core $180^{\circ}$ bending magnet, and a quadrupole. An example of the beam envelope simulation by the OptiM code [4] is shown in Fig.2.

The gun and collector are pumped by two 20-1/s ion pumps mounted inside the terminal. Four standard "crosses" are installed for pumping the beam line. Each includes a 50-1/s ion pump and a titanium sublimation pump. The crosses are also equipped by wire scanners used for beam tuning at low beam currents.

\section{COMMISSIONING}

The Pelletron was assembled in earlier 2001, and a terminal voltage up to $6 \mathrm{MV}$, without tubes installed, was easily achieved. After installing and conditioning the tubes, a work with beam started.

\subsection{Tube conditioning}

When a high voltage is applied to tubes for the first time, partial or full discharges through a tube occur. The energy stored in the electrostatic capacitance of the terminal is about $3 \mathrm{~kJ}$ at nominal energy. During strong discharges the energy can cause (and did cause) significant damages both to an equipment and to the electric strength of the tubes. To make the process of an increasing the terminal voltage (so-called tube conditioning) more safe, the tube modules were conditioned separately. A special external rod allows electrical connection of neighboring separator boxes without losing of the insulating gas, so the voltage can be applied to a single section between separation boxes (i.e. to two tube modules on each side only). In this case, the

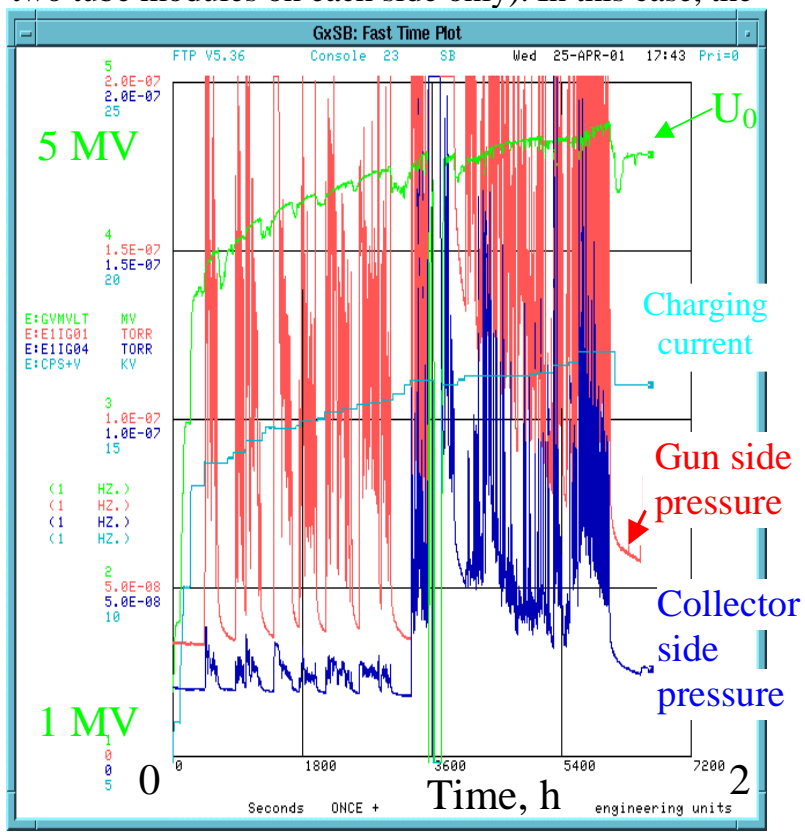

Figure 3. Conditioning of the acceleration tube after exposing it to atmosphere. The terminal voltage, a value proportional to the charging current, and the levels of vacuum pressure for the tubes are shown. The true value of $U_{0}$ is believed to be $6 \%$ lower than shown. 
energy stored at the same electric field gradient decreases dramatically (by 25 times). The voltage on each section went easily to 0.6- $0.8 \mathrm{MV}$, but further conditioning up to $1.1-1.2 \mathrm{MV}$ took about 10 hours per section. When the voltage was applied to the whole tubes, a value of 4.3 MV was achieved in several hours. Further multiple attempts failed to reach the level of $5 \mathrm{MV}$, standard for this tube length. The boundary of a stable operation, 4.2- 4.3 MV is below the voltage necessary for the REC, and NEC will replace the tubes during the year 2001.

After the initial conditioning, the stabilization system works in accordance to specifications (at $U_{0}<4.3 \mathrm{MV}$ ); the total measured voltage deviation is within $\pm 0.3 \mathrm{kV}$.

A conditioned tube does not experience discharges until the voltage reaches the boundary of stable operation. If a tube is exposed to atmosphere, it needs to be conditioned during several hours (Fig.3).

\section{Work with a beam}

Work with beam began at an electron energy of 0.5 $\mathrm{MeV}$, without filling the tank with $\mathrm{SF}_{6}$. Successful passing of a several microampere beam to the collector proved that all elements worked properly. An important result was an absence, even at this low energy, of any beam position jitter that could be caused by AC magnetic fields from motors or from the electric grid. The beam was then fully recirculated at the energy of $4.1 \mathrm{MeV}$.

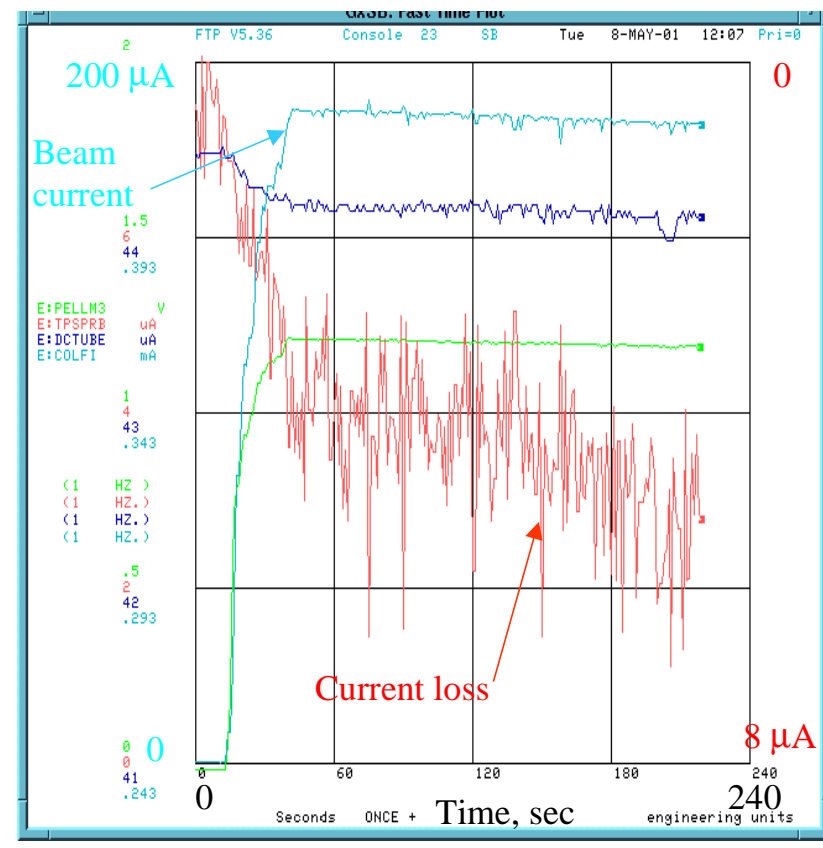

Figure 4. The beam recirculation. The beam current and the current loss are measured by a collector power supply and as a change in the needle current, correspondingly. The electron energy is $4.1 \mathrm{MeV}$, the anode voltage is 25 $\mathrm{kV}$, the collector voltage is $4 \mathrm{kV}$.

The most complicated part of the initial beam tuning is its steering to the collector. While the beam location in the transport line is reliably measured by wire scanners at a beam current of more than $50 \mathrm{nA}$, its position in the deceleration tube can only be determined by indirect measurements. For this purpose, changes in currents of the anode power supply $\Delta I_{a}$, needle $\Delta I_{\text {needle }}$, and tube resistive dividers $I_{a c c}, I_{d e c}$ are used. The value of $\Delta I_{a}$, is always equal to the part of the beam current that does not reach the collector, because the lost current can return to the cathode through the anode power supply only. If the beam is lost in the beam line, $\delta I=\Delta I_{a}=\Delta I_{\text {needle }}$, and the resistive divider currents are unchanged. The losses occurring in the lowest section of the deceleration tube results in a difference between $I_{a c c}$ and $I_{d e c}$. Changes in these currents are always negative, that can be explained by a high secondary electron emission coefficient $(\sigma>1)$. When the beam hits an upper portion of the deceleration tube, the resistive divider currents decrease evenly (resistor chains are connected at each separator box). In this position, the change of the needle current becomes much larger than the beam loss itself; typically, $\Delta I_{\text {needle }} / \Delta I_{a} \sim 20 \div 30$. Since the secondary electron emission, causing this effect, can significantly redistribute the potential along the tubes, the regime was found unstable and easily resulted in breakdowns. The next step is the beam coming to the collector anode, where $\Delta I_{a} \gg \Delta I_{\text {needle }}$. Finally, a minimum in the anode current shows that the beam has reached the collector. Beam current larger than several microamperes is measured by a current monitor installed in the transport line. The maximum beam current of $400 \mu \mathrm{A}$ has been seen.

The progress with recirculation has been delayed by multiple repairs of electronics, primarily at the terminal level, and by an incident when a beam burned a hole in a bellow inside the bend. In the coming shutdown, we will improve the electronics robustness, will install a system of a fast gun shut-off, and will add interlocks to prevent vacuum incidents.

\section{CONCLUSION}

A set-up consisting of a 5-MV Pelletron and a simlified beam line has been designed, manufactured, and installed at Fermilab in 1999-2001. An algorithm for establishing a beam recirculation has been found. Recirculation of a 400 $\mu \mathrm{A}, 4.1 \mathrm{MeV}$ beam has been demonstrated. To move further, reliability of the electronics in the high voltage terminal and protection systems shall be improved. Stable operation with parameters listed in Table 1 is expected by the end of 2001.

Authors are thankful to the all members of the REC group and to the NEC team.

\section{REFERENCES}

[1] S.Nagaitsev et al., NIM A441(2000), 241

[2] A.C.Crawford et al., NIM A435 (1999), 339

[3] National Electrostatic Corporation, 7540 Graber Road, Middleton, WI 53562, U.S.A., www.pelletron.com

[4] LDBS Co., http://host.sybercom.net/users/ld 